\title{
Size and shape of crystallites and internal stresses in carbon blacks
}

\author{
T. Ungár ${ }^{1)}$, J. Gubicza $^{2)}$, G. Tichy ${ }^{2)}$, C. Pantea ${ }^{3)}$ and T.W. Zerda ${ }^{3)}$
}

1) Department of General Physics, Eötvös University Budapest, Hungary

2) Department of Solid State Physics, Eötvös University Budapest, Hungary

3) Department of Physics, Texas Cristian University, Fort Worth, Texas, USA

\begin{abstract}
The effect of graphitization and pressure treatment on the microstructure and internal stresses of carbon blacks is studied by X-ray diffraction peak profile analysis. The Fourier transforms of the experimental line profiles are fitted by theoretical functions based on the model of the microstructure. In this model the crystallites are ellipsoids with log-normal size distribution. It was found that in raw carbon blacks crystallites have lateral dimensions twice the size in the hexagonal $c$ direction. During graphitization process caused by heat treatment the crystallites grow and their shapes becomes more spherical. The pressure has no effect on the size and shape of crystallites. The internal strains and stresses in the crystallites are determined from the X-ray diffraction profiles by the Griffith-model. It was established that the internal stresses increase due to pressure treatment while they are reduced by graphitization.
\end{abstract}




\section{Introduction}

Carbon black is generally used as a filler in rubber production to modify the mechanical properties of the tire. Although the smallest indivisible unit of carbon black is the aggregate, in the TEM images aggregates appear to be formed by spherical particles, which are fused together. Aggregates connect through Van der Waals forces into networks called agglomerates [1]. Internal structure of aggregates is not well understood. Graphite-like, quasicrystalline domains, in which basal planes are parallel but angularly distorted and the spacing between the layers is different from that of pure graphite, have been detected in carbon black particles [1]. Biscoe and Warren identified those structures as intermediate between crystalline and amorphous materials [2]. Hereafter, the turbostratic quasi-crystalline domains are referred to as graphitic crystallites or nanocrystallites. Due to the lack of threedimensional ordering, this nomenclature is not rigorously correct, but appears to be generally accepted in the scientific literature [1,2-5]. Warren and Bodenstein [6] computed diffraction patterns of carbon blacks assuming turbostratically arranged layer structures in which blocks of parallel layers normal to the $c$ direction have small but finite size: $\mathrm{L}_{\mathrm{c}}$. The numerically calculated diffraction pattern for $L_{c}=1.38 \mathrm{~nm}$ and the lateral size of the blocks $L_{a}=2.0 \mathrm{~nm}$ with $\mathrm{CuK} \alpha$ radiation is reproduced in Fig. 1 [6]. It is important to note that in this model calculation strain was not included.

The structure of carbon black particles and their surface properties are important for many reasons. Different sites present on the surface determine reinforcing properties of carbon black in rubber [7]. Energy of interaction between the polymer and carbon black depends on nature and population of different sites. The most energetic sites, which very effectively adsorb polymers, are usually identified as crystallite edges. Crystallite flat surfaces and amorphous carbon present on the surface are considered less energetic sites. Thus to characterize reinforcing properties of carbon black it is necessary to determine the fraction of 
the surface occupied by amorphous carbon and estimate the size of the crystallites and their surface density. Relative concentration of amorphous carbon can be evaluated from Raman measurements [3]. Energies of adsorption of different adsorption sites and their relative populations have recently been evaluated by gas adsorption technique [8]. The sizes of graphitic crystallites have been estimated from X-ray diffraction [5,9], neutron scattering [10], Atomic Force Microscopy [11], and Raman spectroscopy measurements [3-5]. Those studies showed that the average size of crystallites, $\mathrm{L}_{\mathrm{a}}$, is similar for all commercial carbon black grades produced in furnace reactors and roughly equal $2.3 \mathrm{~nm}$. This was a surprising result because different grades have completely different rubber reinforcing properties.

Small crystallites are not energetically favorable structures. One possible explanation for their presence in carbon blacks that large strains are developed in production, i.e. during aggregate growth at high temperatures and also during water quenching, when temperature of carbon black is rapidly reduced from about $1600{ }^{\circ} \mathrm{C}$ to about $800{ }^{\circ} \mathrm{C}$. Strain is relieved when larger structures brake to form smaller crystallites. Characterization of strain remaining in the crystallites is an important problem, which so far has not been addressed. Strain can change bond lengths and angles, and thus affect surface potential and in turn reinforcing properties of carbon blacks. Reinforcing properties of carbon black are also expected to be a function of crystallite size distribution. Carbon black with uniform crystallites that all have similar sizes is expected to have more homogenous surface potential than carbon black exhibiting a wide distribution of sizes.

In this paper the effect of graphitization and pressure treatment on (a) the size distribution and (b) the shape of crystallites as well as (c) the strain in the crystallites of carbon blacks is studied by X-ray diffraction profile analysis. The values of the average crystallite sizes determined by X-rays were compared with those obtained from Raman measurements. 


\section{Experimental}

\subsection{Sample preparation}

The grade of carbon black selected for this study is N774 obtained in furnace reactors. It is mainly used in tire carcasses. This carbon black was heat treated for 20 minutes at $2700^{\circ} \mathrm{C}$ ( labeled as G, graphitized). To avoid oxidation the chamber of the induction furnace was purged and filled with gaseous nitrogen. After the heat-treatment the samples were allowed to cool down slowly to room temperature. High pressure experiments were conducted at room temperature inside a piston cylinder at the pressure of $2.5 \mathrm{GPa}(25,000 \mathrm{bar})$. The samples were allowed to remain compressed for about 20 minutes. The raw carbon blacks exposed to high pressure are denoted as $\mathrm{P}$, those which first underwent heat treatment are called PG.

\subsection{X-ray diffraction technique}

The diffractograms for carbon black specimens were measured by a Philips X'pert diffractometer using $\mathrm{Cu}$ anode and pyrolitic graphite secondary monochromator. The details of the profiles were measured in a special double crystal, high-resolution diffractometer with negligible instrumental broadening. The overlapping peaks were better separated in these measurements due to the absence of the $\mathrm{K}$ alpha doublet. In the high resolution diffractometer a fine focus rotating cobalt anode (Nonius FR 591) was operated as a line focus at $36 \mathrm{kV}$ and $50 \mathrm{~mA}(\lambda=0.1789 \mathrm{~nm})$. The symmetrical 220 reflection of a Ge single crystal was used for monochromatization of the beam. The profiles were registered by a linear position sensitive gas flow detector, OED 50 Braun, Munich. Seven diffraction peaks indexed as 0002, 1010, $10 \underline{11}, 0004,11 \underline{2} 0,11 \underline{2} 2$ and 0006 were recorded. $10 \underline{10}$ and $10 \underline{11}$ as well as $11 \underline{20}, 11 \underline{2} 2$ and 
0006 overlap and were separated by fitting procedures. The most intensive five profiles 0002 , $10 \underline{10}, 10 \underline{1} 1,0004$ and $11 \underline{20}$ were used in the evaluation method.

\subsection{Raman spectroscopy}

Complementary data on average lateral sizes of crystallites were obtained from Raman scattering measurements. In addition, Raman studies allowed us to estimate the relative concentration of amorphous carbon on the surface of carbon black aggregates. Raman spectra were obtained using a home-made spectrometer equipped with a confocal microscope. To avoid laser heating carbon blacks were placed inside a groove in a steel disk spinning at a speed of $3000 \mathrm{rev} / \mathrm{min}$. The average size of nanocrystallites can be determined in terms of changes in position, width and intensity ratio of two Raman peaks observed at $1345 \mathrm{~cm}^{-1}$ (the disordered, or d peak) and $1575 \mathrm{~cm}^{-1}$ (the graphite, or g peak). The shape of those peaks depends on the crystallite sizes. When the crystallites become larger, the peaks become narrower, their maxima move to higher frequencies and the intensity of the g peak systematically increases in comparison with the $d$ peak. Using the empirical formula found by Tuinstra and Koenig [12]

$$
\mathrm{L}_{\mathrm{a}}=4.35 \mathrm{Ig}_{\mathrm{g}} / \mathrm{I}_{\mathrm{d}}[\mathrm{nm}]
$$

it is possible to evaluate $\mathrm{L}_{\mathrm{a}}$, the lateral size of the crystallites. Amorphous carbon is observed as a broad peak centered at about $1530 \mathrm{~cm}^{-1}$. Its intensity relative to that of the $\mathrm{d}$ and $\mathrm{g}$ peaks is an estimate of the relative concentration of amorphous carbon on the surface of carbon black aggregates [3]. 
A numerical procedure has been worked out for fitting the Fourier transform of the experimental diffraction profiles by the product of the theoretical functions of size and strain Fourier transforms given in Refs. [13-15]. In the calculation of the theoretical functions the carbon black crystallites are modeled by ellipsoids of revolution because former Raman spectroscopy investigations have shown that the carbon black crystallites are non-spherical flat discs [3-5]. The shape of the crystallites are described by the ellipticity, $\varepsilon$, the ratio of the diameters $D_{c}$ and $D_{a}$, parallel and perpendicular to the hexagonal axis of crystallites. It is assumed that ellipticity, $\varepsilon$, is the same for all crystallites, and that the crystallite size distribution density function, $\mathrm{f}(\mathrm{x})$, is log-normal. It is also assumed that in the crystals the lattice distortions are caused by dislocations. According to this model of the microstructure, the theoretical Fourier transforms of the diffraction profiles are calculated. These profiles are fitted to the Fourier coefficients of the experimental intensity profiles by the Multiple Whole Profile (MWP) fitting method [13-15]. The method has the following steps: i) the Fourier coefficients of the measured physical profiles have been calculated, ii) the theoretical Fourier coefficients of the size and strain profiles were calculated, (iii) the experimental and the calculated Fourier coefficients were compared by the least squares method. From the MWP procedure the following parameters of the microstructure can be obtained for the carbon blacks: (i) the median, $m_{a}$, of the size distribution of diameters of crystallites perpendicular to the hexagonal axis, (ii) the variance, $\sigma$, of the log-normal crystallite size distribution function, (iii) the ellipticity of the crystallite shape, $\varepsilon$, (iv) the density, $\rho$, and (v) the arrangement parameter, M, of dislocations. Further details of the fitting procedure are given elsewhere [13$15]$.

For the carbon black powder samples strong overlap of the diffraction profiles has been observed. The overlapping peaks have to be separated since the present evaluation method is 
worked out for individual profiles. Background subtraction and the separation of overlapping peaks are carried out in one step. Two or more analytical functions, usually of PearsonVII or Pseudo-Voigt type plus a linear background are fitted to the overlapping peaks. In the next step the unwanted fitted peaks together with the linear background are subtracted leaving the targeted peak free of overlap and background. The separated profiles are taken as individual diffraction profiles in the evaluation procedures. Due to very large strains and nanometer size crystallites peak broadening of the carbon black specimens is about two orders of magnitude larger than the instrumental effects. For this reason no instrumental corrections were carried out.

\section{Results and discussion}

\subsection{Average size, size distribution and shape of crystallites}

Powder diffraction patterns for the initial N774 and the graphitized G774 samples are shown in Fig. $2 \mathrm{a}$ and $2 \mathrm{~b}$, respectively. It can be seen that after heat treatment at $2700{ }^{\circ} \mathrm{C}$ the peak profiles become narrower. Note that in Fig. $2 \mathrm{~b}$ the intensity is scaled logarithmically in order to show the details of the peaks at lower intensities. The diffractograms show strong overlap of the peaks for both samples. The overlapping peaks were measured also by a special high resolution double crystal diffractometer and were separated by the method described in section 3 . The median, $m_{a}$, and the variance, $\sigma$, of the size distribution of the crystallite diameter parallel to the hexagonal basal plane, the ellipticity of the crystallite shape, $\varepsilon$, and the density, $\rho$, of dislocations are determined by the MWP numerical fitting procedure [13-15] and are listed in Table 1. The average lateral size values of the crystallites parallel to the hexagonal basal plane, $\mathrm{L}_{\mathrm{a}}$, have been determined from the Raman spectra and are listed in

Table 1. Comparing these values to the median diameter data, $m_{\mathrm{a}}$, obtained from X-ray 
analysis it is seen that the two techniques give similar results. The values corresponding to the specimen compressed after graphitization are not given in Table 1 since pressure did not change the microstructural parameters, compared to the values of the graphitized specimen.

The log-normal size distribution density functions, $f(x)$, calculated from the $m_{\mathrm{a}}$ and $\sigma$ values are plotted in Fig. 3. The figure shows that the graphitization heat treatment shifts $f(x)$ to considerably higher size values and due to this heat treatment the size distribution function becomes very narrow. The increase of the grain size of carbon blacks due to graphitization has been observed before by Raman, TEM, AFM, and also by X-ray diffraction using the Scherrer method [9-11]. These techniques proved that at elevated temperatures crystallites grow both in the lateral and vertical directions. At the same time, it can also be seen from Table 1 and Fig. 3 that the application of pressure does not effect the size distribution, neither before, nor after graphitization. Our previous neutron scattering and Raman in situ highpressure studies on carbon blacks indicated that under pressure the turbostratic structures become organized and carbon-carbon bonds approach values typical for a graphite crystal [4]. The current study was conducted after the pressure treatment and since we did not observe any significant changes in the crystallite sizes we concluded that the system returned to its original configuration. As can be seen from Table 1, the concentration of amorphous carbon is reduced after graphitization while after pressure treatment concentration of amorphous carbon either slightly decreased or remained unchanged.

Approximating the crystallites by ellipsoids, the median of the diameters of the crystallites perpendicular to the hexagonal basal plane is denoted by $\mathrm{m}_{\mathrm{c}}$. This measures the stacking heights of graphitic nanocrystallites in the hexagonal $c$ direction. The value of $\varepsilon=m_{\mathrm{c}} / m_{\mathrm{a}}$ is between 0.5 and 1.0 , in good agreement with previous studies, i.e. the heights of the crystallites vary between $50 \%$ and $100 \%$ of their corresponding lateral sizes. In the untreated samples the lateral sizes of crystallites $\left(L_{\mathrm{a}}\right.$ or $\left.m_{\mathrm{a}}\right)$ are usually twice the size of the 
stacking height of the layers, $m_{\mathrm{c}}$. But after heat treatment the lateral and vertical dimensions become similar, compare Table 1 . The pressure has no effect on the shape of the crystallites. The full widths at half maximum (FWHM) as a function of the reciprocal space coordinate, $\mathrm{K}=2 \sin \theta / \lambda$, where $\theta$ and $\lambda$ are the diffraction angle and the wavelength of $\mathrm{X}$-rays, are shown in the Williamson-Hall plot [16] in Fig. 4. It can be seen that in the case of the initial state (N774) and compressed (P774) specimens the $h k 0$ and $h 0 l$ type of reflections are well separated into two groups, where the latter have considerably higher values of FWHM. This indicates that the crystallites of these two specimens have smaller dimensions in the $c$, than in the lateral direction in good correlation with the quantitative evaluation of crystallite ellipticity listed in Table 1. The Williamson-Hall plot also shows that the FWHM values of the different reflections for the two different specimens are in the same heights. This means that the crystallite size values in the two different crystallographic directions are very similar for the two specimens. The FWHM values corresponding to the $h k 0$ and $h 0 l$ type reflections are connected by schematic curves. The slopes of these cuvees indicate qualitatively the average lattice strains in the particles in the $h k 0$ and $h 0 l$ specific directions, latter showing out of the basal plane. It is obvious from the figure that the strains are qualitatively stronger in the $c$ direction as compared to the lateral directions. The FWHM values of the graphitized specimen (G774) are much lower than for N774 or P774 samples indicating the increase of the crystallite size during heat-treatment. The slope of the curve connecting the FWHM values is decreasing due to the decrease of the lattice strains.

\subsection{Internal strains and stresses obtained from the formal values of dislocation densities}

When nanoparticles aggregate, welding occurs between contacting surfaces due to strong adhesion [17]. The same adhesion interaction produces large strains and stresses where the particles are connected by necking contacts [18]. In the necking contacts the particles are 
welded together along the necking surface. A schematic drawing of three agglomerating spheres is shown in Fig. 5a. The opening between the particles has a free surface with an excess surface energy. The equilibrium configuration of the necking surfaces and the free surfaces between the particles is governed by the energy balance between the excess free surface energy and the work produced by the adhesion interaction. Latter pushes the particles toward each other in order to increase the necking surface and decrease the free surface. The contour lines indicating the necking- and the free surfaces can bee seen in Fig. 5b. The opening between the particles together with the welded region, ranging along the necking surface, can be considered physically equivalent to the tip of a crack in a solid. Griffith has shown that a strained crack tip produces inhomogeneous strain and stress fields which can be given as the deformation field produced by a superdislocation with Burgers vector $\boldsymbol{B}$ [19]:

$$
\boldsymbol{B}=\frac{\mathrm{P}_{\mathrm{o}}(1-v)}{\mu} 2 \mathrm{~L},
$$

where $\mathrm{P}_{\mathrm{o}}$ is the stress acting in the direction parallel to $\boldsymbol{B}$ on the free surfaces of the opening, $\mu$ and $v$ are the shear modulus and the Poisson's ratio of the material and $L$ is the particle diameter. On the basis of simple considerations the formal dislocation density, $\rho$, provided by the standard line profile analysis used here, can be related to $\boldsymbol{B}$ as:

$$
\rho=\frac{B}{b L^{2}},
$$

where $b$ is the absolute value of the Burgers vector of crystal dislocations. From (2) and (3):

$$
\mathrm{P}_{\mathrm{o}}=\frac{\mu}{2(1-v)} \rho b L
$$


The magnitude of internal stresses is characterized by $\mathrm{P}_{\mathrm{o}}$ calculated from the formal dislocation density, $\rho$, obtained from the fitting procedure and using the shear modulus of graphite $\mu=4.8 \mathrm{GPa}[20]$. The absolute value of the Burgers vector of crystal dislocations is taken as the arithmetic average of $a$ and $c$ lattice parameters: $0.5 \mathrm{~nm}$. The crystallite size, $L$, in eq. (4) is calculated as the average of $m_{\mathrm{c}}$ and $m_{\mathrm{a}}$ diameters. The internal strains are calculated from eq. (4) as: $\rho b L$. The values of the Griffith strain and stress are listed in Table 1. The magnitude of strains and stresses decreases slightly with graphitization but increases after pressure treatment.

\section{Conclusions}

In the as prepared state of carbon black of N774 grade the crystallites have anisotropic shape: in the lateral directions the crystallite size is about twice as large as in the hexagonal $c$ direction. The shape anisotropy has been modeled by ellipsoids of revolution. Graphitization increases the average crystallite size and crystallite shape tends toward spheroidisation. The microstresses have been evaluated in terms of crystal dislocations. Since, however, the presence of dislocations in carbon black has not yet been verified experimentally, the formal dislocation density has been converted to stresses prevailing between crystallites attached to each other by necking. The conversion has been carried out by using the Griffith model of stresses in strained cracks. It is found that compression induces heterogeneous internal microstresses, whereas, heat treatment slightly decreases these stresses. Compression does not change the crystallite size or shape. 
Acknowledgements

The authors are grateful to the Hungarian National Science Foundation, OTKA, Grant Nos. T046990 and F047057. 
References

1. J.B. Donnet, A. Voet, Carbon black: Physics, chemistry and elastomer reinforcement, Dekker, New York, 1976, p. 50.

2. J. Biscoe, B.E. Warren, J Appl Phys, 13 (1942) 364.

3. T.W. Zerda, W. Xu, H. Yuang, M. Gerspacher, Rubber Chem Technol, 71 (1998) 26.

4. T.W. Zerda, W. Xu, A. Zerda, Y. Zhao, R.B. Von Dreele, Carbon, 38 (2000) 355.

5. T. Gruber, T.W. Zerda, M. Gerspacher, Carbon, 32 (1994) 1377.

6. B.E. Warren, P. Bodenstein, Acta Cryst, 18 (1965) 282.

7. M. Gerspacher, C.P. O’Farrell, W.A. Wampler, Rubber World, 212 (1995) 26.

8. A. Schroder, M. Kluppel, R. Schuster, J. Heidberg, Proc. ACS Rubber Division Meeting, Dallas, April 2000, Kautschuk, Gummi, Kunststoff 5 (2001) 260.

9. T.W. Zerda, C. Pantea, J. Qian, T. Ungár, Proc. MRS Fall meeting, Symp: Filled and Nanocomposite Polymer Materials, eds. Hjelm RP, Nakatani AI, Gerspacher M, Krishnamoorti R. Boston, U.S.A. 2000, KK6.4.1-8.

10. R. Hjelm, W.A. Wampler, P.A. Seeger, M. Gerspacher, J Mater Res 9 (1994) 3210.

11. W. Xu, T.W. Zerda, H. Raab, D. Goritz, Carbon, 35 (1997) 471.

12. F. Tuinstra, J.L. Koenig, J Chem Phys 53 (1970) 1126.

13. T. Ungár, J. Gubicza, G. Ribárik, A. Borbély, J. Appl. Cryst. 34 (2001) 298.

14. G. Ribárik, T. Ungár, J. Gubicza, J. Appl. Cryst. 34 (2001) 669.

15. T. Ungár, J. Gubicza, G. Ribárik, C. Pantea, T. W. Zerda, Carbon, 40 (2001) 929.

16. G. K. Williamson, W. H. Hall, Acta metall. 1 (1953) 22.

17. Y. Yao, A. Thölén, Mater. Characterisation, 44 (2000) 441.

18. F. Wakai, F. Aldinger, Acta Mater, 51 (2003) 641.

19. A.A. Griffith, Phil. Trans. R. Soc, A221 (1920/21) 163.

20. K. Maslov and V. K. Kinra, Mater. Sci. Eng. A, 367 (2004) 89. 
Figure captions:

Figure 1. Computed diffraction pattern of carbon blacks for the lateral size $\mathrm{L}_{\mathrm{a}}=2.0 \mathrm{~nm}$ and the size of $\mathrm{L}_{\mathrm{c}}=1.38 \mathrm{~nm}$ in the hexagonal $c$ direction with $\mathrm{CuK} \alpha$ radiation. The dashed curves give the Compton modified and the independent unmodified scattering per atom. Courtesy of B. E. Warren and P. Bodenstein [6].

Figure 2. The powder diffractogramms for the N774 carbon black specimen in the initial state (a) and for the specimen G774 after annealing at $2700{ }^{\circ} \mathrm{C}$ (b). The intensity in logarithmic scale in 1(b) shows details of low intensity peaks.

Figure 3. Crystallite size distribution functions for the as-received specimen N774 (initial state), after heat treatment at $2700{ }^{0} \mathrm{C}$ (graphitized), after pressure treatment at $2.5 \mathrm{GPa}$ (compressed), and graphitized carbon black after pressure treatment (dotted line).

Figure 4. The full widths at half maximum (FWHM) versus the reciprocal space coordinate, K (Williamson-Hall plot) for the as-received specimen (initial state), the graphitized and compressed samples.

Figure 5. (a) Schematic drawing of three touching particles. (b) The contours of the opening between particles (1) and (2). The symbol T stands for a superdislocation producing the inhomogeneous strains and stresses due to the strong cohesion pressing the particles together. The upward arrow represents the Burgers, $\boldsymbol{B}$, vector of the superdislocation according to the Griffith model of strained cracks [19]. 
Table 1. Lateral crystallite size, $\mathrm{L}_{\mathrm{a}}$, and relative content of amorphous carbon, $\mathrm{I}_{\mathrm{a}} / \mathrm{I}_{\text {total }}$, obtained from Raman spectroscopy; the median, $\mathrm{m}_{\mathrm{a}}$, and the variance, $\sigma$, of the distribution of the crystallite-diameters parallel to the hexagonal basal plane, the ellipticity of the crystallite shape and the Griffith strain and stress values determined by X-ray diffraction for carbon blacks at various histories of temperature and pressure treatments.

\begin{tabular}{|c|c|c|c|c|c|c|c|}
\hline sample & $\varepsilon=\mathrm{D}_{\mathrm{c}} / \mathrm{D}_{\mathrm{a}}$ & $\begin{array}{c}\mathrm{L}_{\mathrm{a}}(\mathrm{nm}) \\
\text { Raman }\end{array}$ & $\mathrm{m}_{\mathrm{a}}(\mathrm{nm})$ & $\sigma$ & $\begin{array}{c}\text { Griffith } \\
\text { strain }\end{array}$ & $\begin{array}{c}\text { Griffith } \\
\text { stress } \\
{[\mathrm{MPa}]}\end{array}$ & $\begin{array}{c}\mathrm{I}_{\mathrm{a}} / \mathrm{I}_{\text {total }} \\
\text { Raman }\end{array}$ \\
\hline $\mathrm{N} 774$ & $0.5(2)$ & $2.3(2)$ & $2.7(5)$ & $0.31(3)$ & $0.027(3)$ & $33(5)$ & 0.24 \\
\hline $\mathrm{P} 774$ & $0.6(2)$ & $2.8(2)$ & $3.6(5)$ & $0.16(2)$ & $0.090(5)$ & $110(10)$ & 0.20 \\
\hline $\mathrm{G} 774$ & $1.2(2)$ & $11.5(5)$ & $11.3(5)$ & $0.02(1)$ & $0.018(3)$ & $22(5)$ & 0.02 \\
\hline
\end{tabular}


Fig.1.

T. Ungár et al.

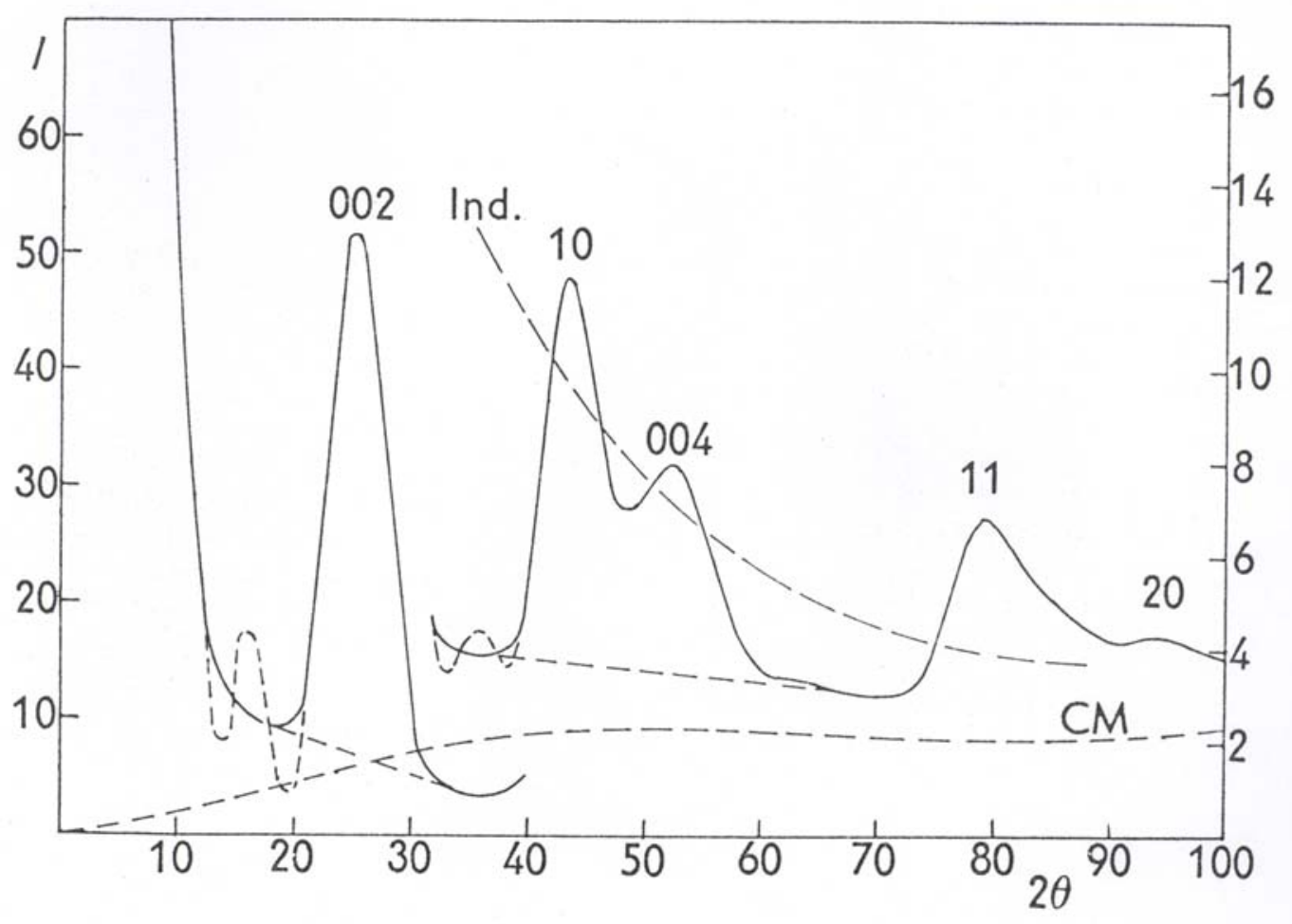


Fig.2.a.

T. Ungár et al.

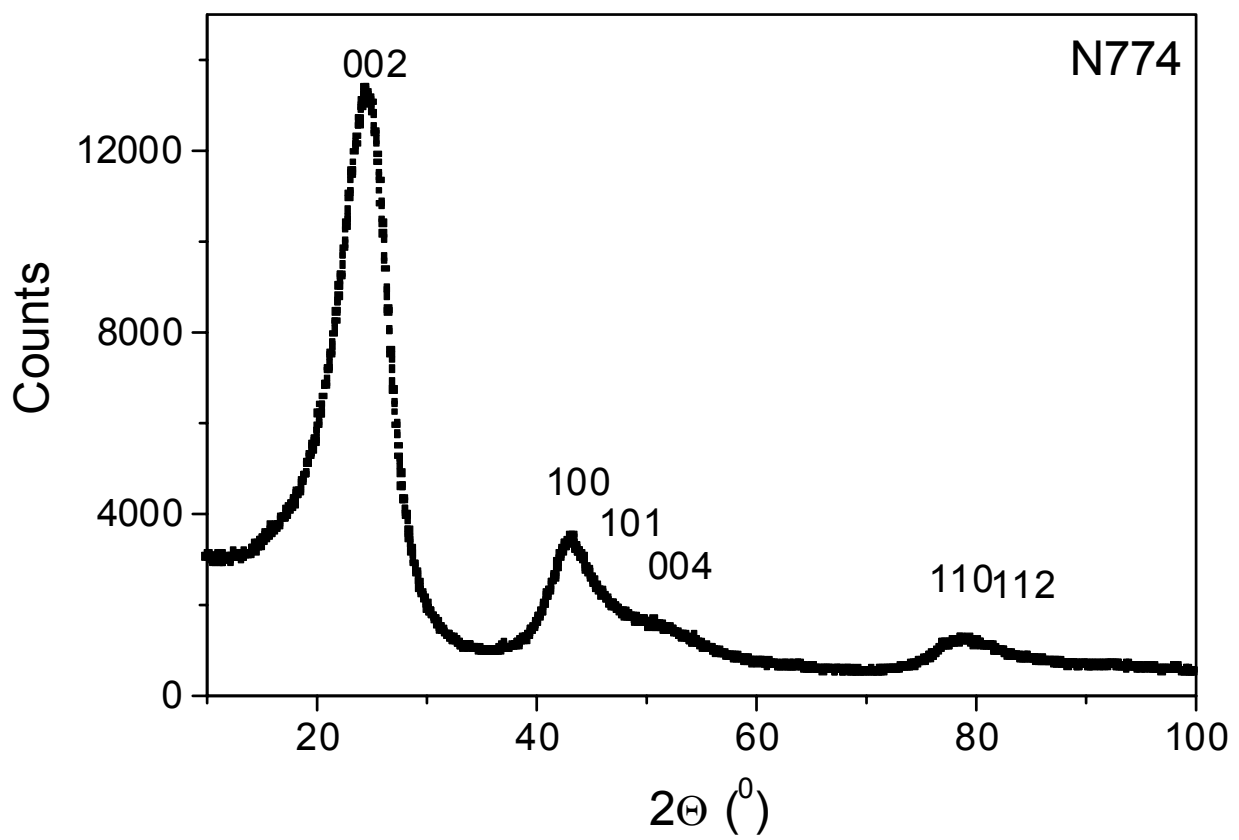


Fig.2.b.

T. Ungár et al.

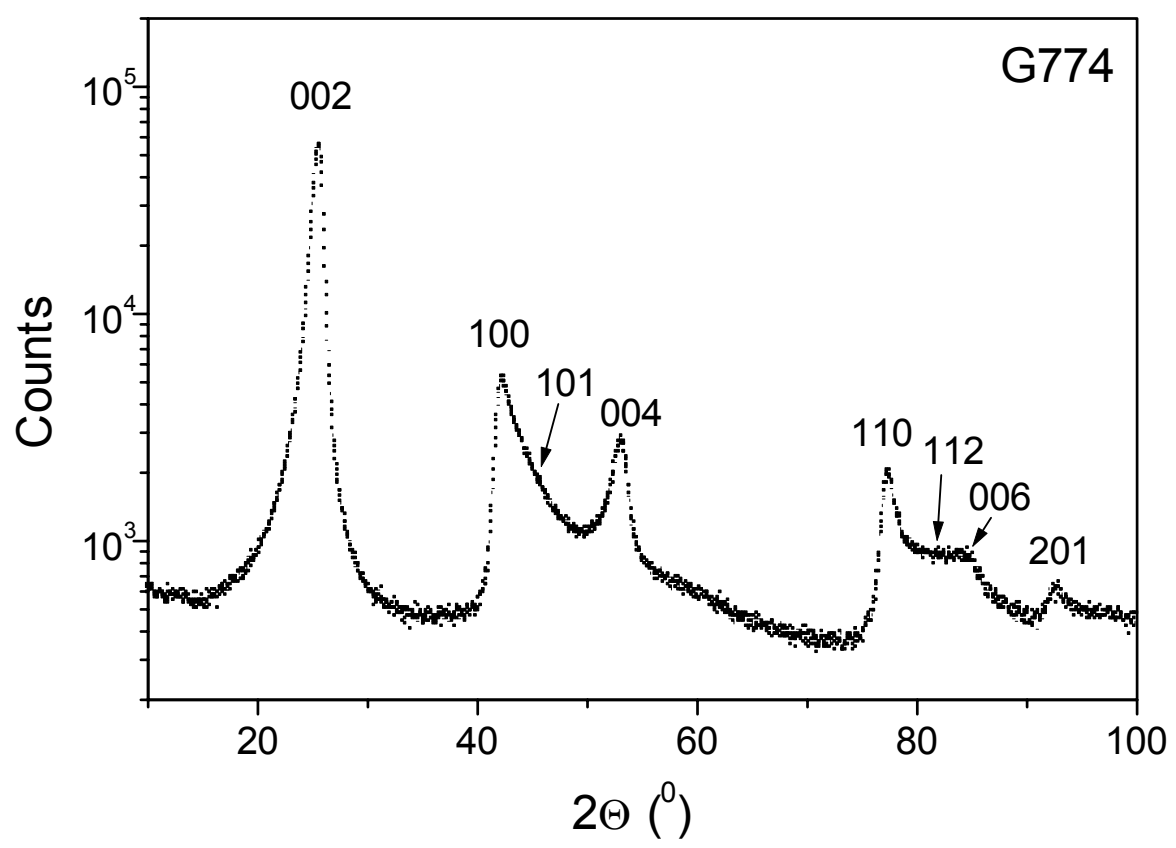


Fig.3.

T. Ungár et al.

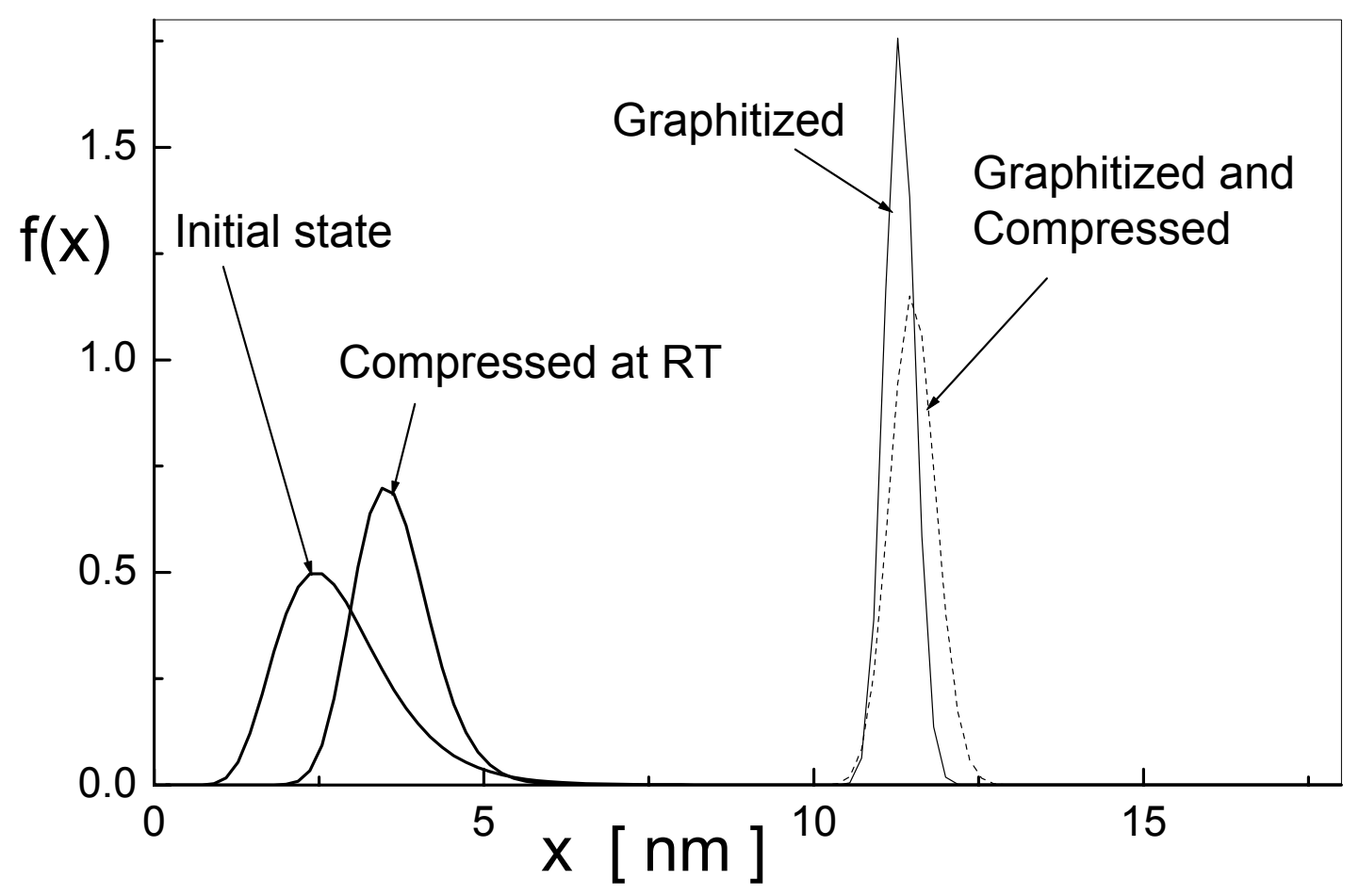


Fig.4.

T. Ungár et al.

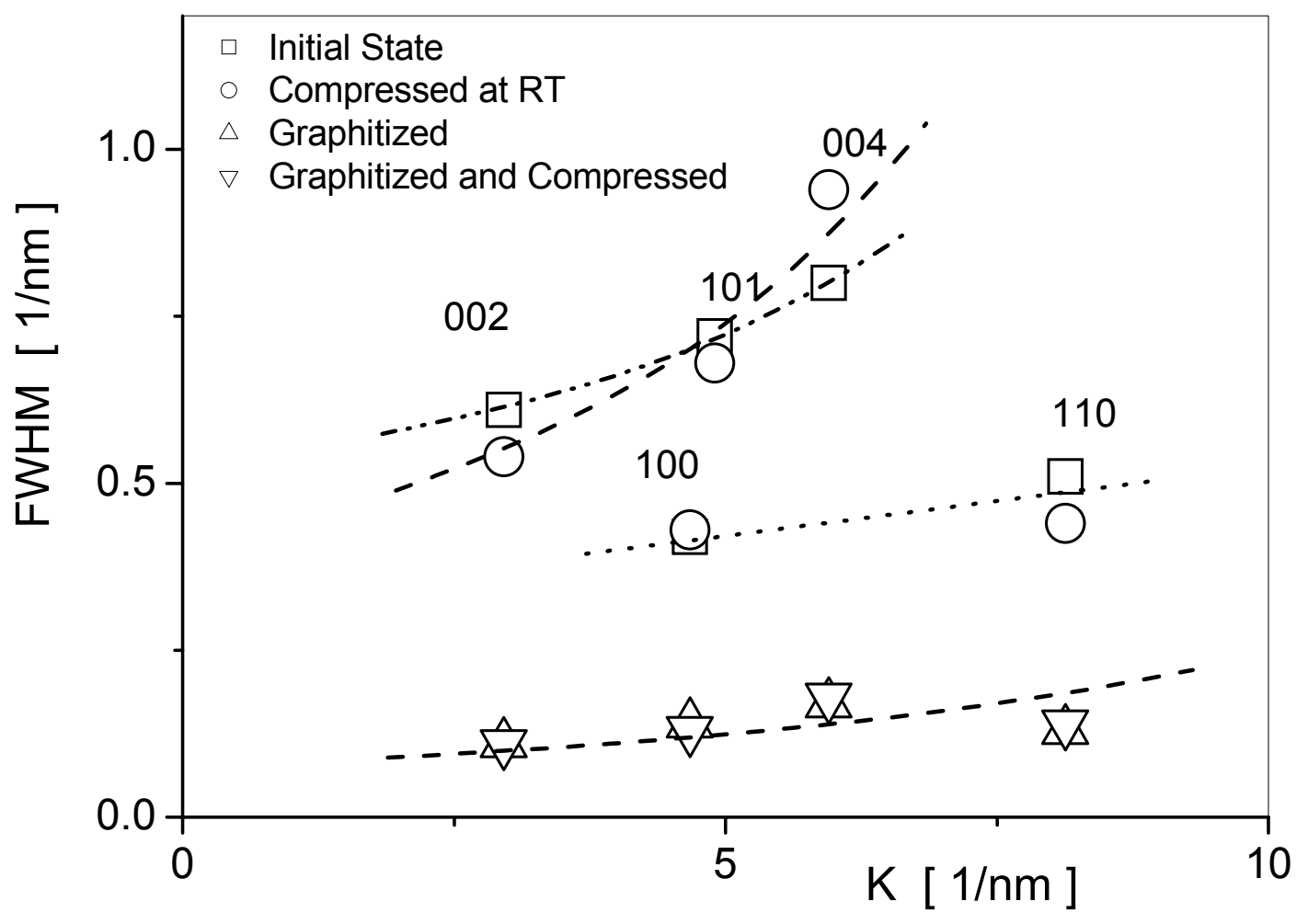


Fig.5.

T. Ungár et al.

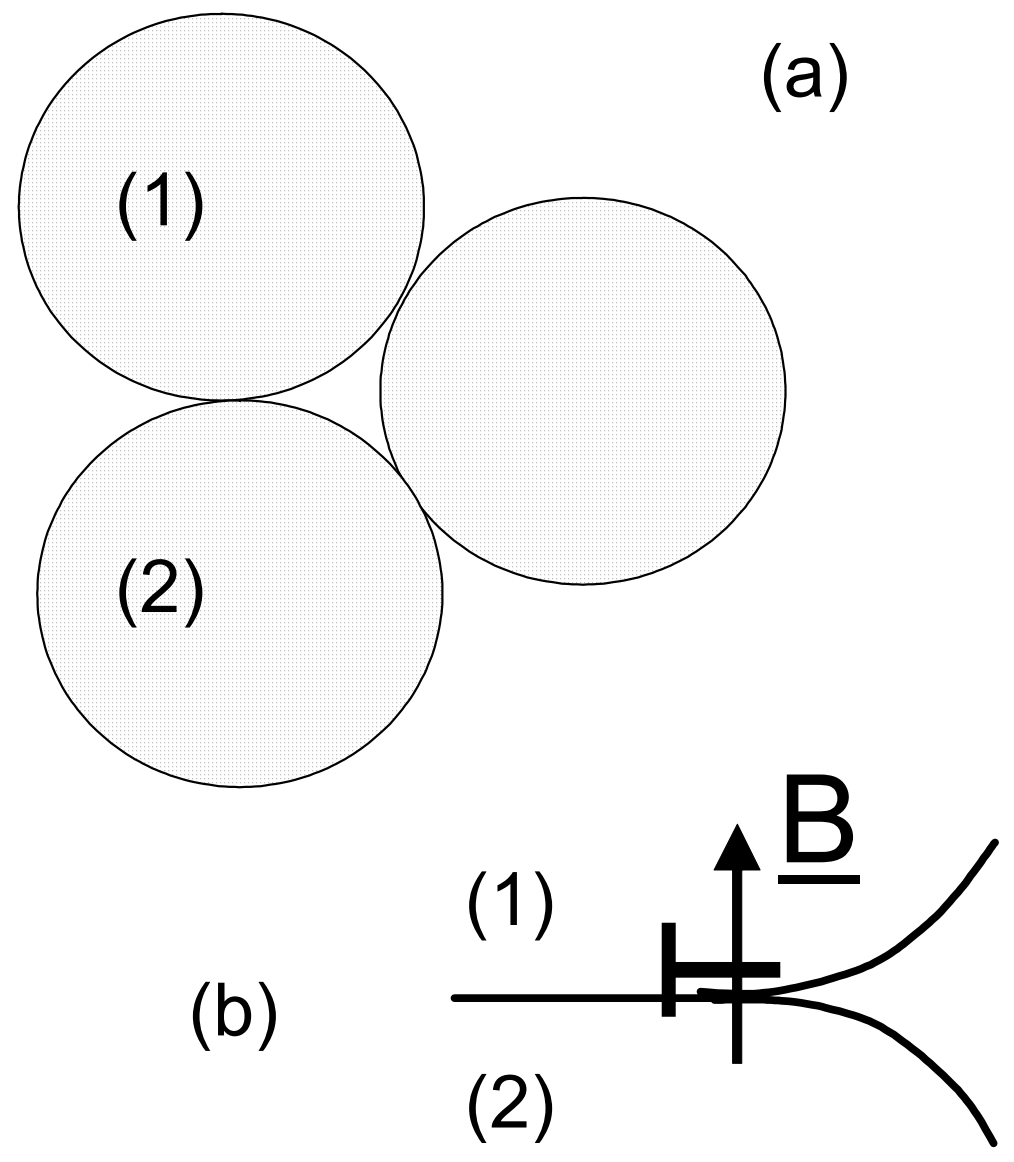

